\title{
Low Voltage Silicon Drift Detector Microanalysis of the Mineral Tourmaline: Examples From the Black Hills, South Dakota
}

\author{
Craig S. Schwandt \\ McCrone Associates, Inc., Westmont, IL, USA
}

The mineral tourmaline is a cyclosilicate belonging to the trigonal crystal system and is notable for its boron content in form of borate anions. Tourmaline is typically a late-stage mineral occurring in pegmatite (coarse-grained granitic rock that was rich in crustal fluids containing lithophile elements during final emplacement). As a result, a significant amount of elemental solid substitution is possible. The general formula with possible substitutents is: $(\mathrm{Ca}, \mathrm{Na})(\mathrm{Al}$, $\left.\mathrm{Fe}^{2+}, \mathrm{Fe}^{3+}, \mathrm{Li}, \mathrm{Mg}, \mathrm{Mn}\right)_{3} \mathrm{Al}_{6} \mathrm{Si}_{6} \mathrm{O}_{18}\left(\mathrm{BO}_{3}\right)_{3}(\mathrm{~F}, \mathrm{O}, \mathrm{OH})_{4}[1,2]$.

New tourmaline occurrences were recently recognized in the Black Hills, South Dakota [3], and their compositions are required in order to understand the petrogenesis of the tourmaline and matrix rock. Traditionally tourmaline was analyzed by electron probe microanalysis (EPMA) conducted with beam energies of at least $15 \mathrm{kV}$. Historically, measurement of boron and oxygen by EPMA was not routine because appropriate wavelength dispersive spectrometer crystals for such light elements were not commonly available. Therefore, EPMA analyses were deemed acceptable based on crystal chemical assumptions. One assumption was that the substitution of aluminum for silicon in the tetrahedral structure positions was very limited and therefore EPMA analyses should be normalized to six silicon atoms. In addition it was also assumed that boron when measured was stoichiometric and the oxygen was determined by difference. Alternatively, [2] although deemed less ideal, was the stoichiometric determination of oxygen based on 29 oxygen atoms per formula unit, with boron determined by difference. Thus, in terms of the accuracy of absolute elemental concentrations tourmaline analyses have been poor. Analyses were therefore only meaningful when conducted using the same protocol.

Given the number of assumptions made using a traditional EPMA approach, the technology advances of the past decade in terms of silicon drift detectors (SDD) [4] now allow for improved microanalysis [5] of tourmaline measuring all major essential structural constituent elements, especially boron and oxygen. However, the analyses must be conducted at low electron beam energy so that the boron X-rays are not swamped by the background produced by many $l$ and $m$ lines from high atomic number elements, and so that due to increased penetration depth boron Xrays do not experience excessive absorption. For example, at $15 \mathrm{kV}$, boron is typically not detected. However, as most of the atomic number, absorption, and fluorescence parameters common to most quantification programs were collected on non-silicate materials at $20 \mathrm{kV}$, they are not appropriate for low energy quantification. Therefore to achieve reasonable results an EPMA-like approach can be used, whereby standard reference materials with a similar matrix to 
the unknown are utilized to conduct standards-based quantification [6]. This approach can be used at low beam energy with some of the newest SDD vendor software or with DTSA-II available from NIST [7].

Tourmaline crystals from two localities in the Black Hills, South Dakota, separated by ten miles are being studied. Plane polarized light images (Figure 1) illustrate the growth zoning preserved at both localities. Over-layered element maps acquired for fifteen minutes with moderate acquisition parameters do not elucidate the zoning present. Point analyses demonstrate minor differences in the ratios of magnesium, iron, aluminum, and the concentration of calcium between the two localities. It is hope that fully quantified results can be obtained by the time of the Conference. However, significant challenges exist with acquiring spectra without either charging issues, or interference from carbon coatings under high vacuum conditions, or under low vacuum conditions which are impacted by secondary fluorescence due to the charge cloud.

\section{References}

[1] WA Deer, RA Howie, and J Zussman, An Intro to the Rock Forming Minerals (1966), Longman, p.90-96.

[2] JJ Papike, Reviews of Geophysics, 25, 7 (1987) p. 1511-1513.

[3] F Griswold, E Peterson, CS Schwandt, J Greenberg, GSA Ann.Meeting (2011), paper 39-21.

[4] DE Newbury, NWM Ritchie, Microsc. Microanal. 18 (Suppl 2), (2012), p. 1010-1011.

[5] DE Newbury, NWM Ritchie, Microsc. Microanal. 18 (Suppl 2), (2012), p. 1004-1005.

[6] N Ritchie, DE Newbury, S Leigh, Microsc. Microanal. 18 (Suppl 2), (2012), p. 1006-1007.

[7] http://www.cstl.nist.gov/div837/837.02/epq/dtsa2/index.html

[8] Samples are part of collaborative research with J Greenberg, Wheaton College, Wheaton, IL.
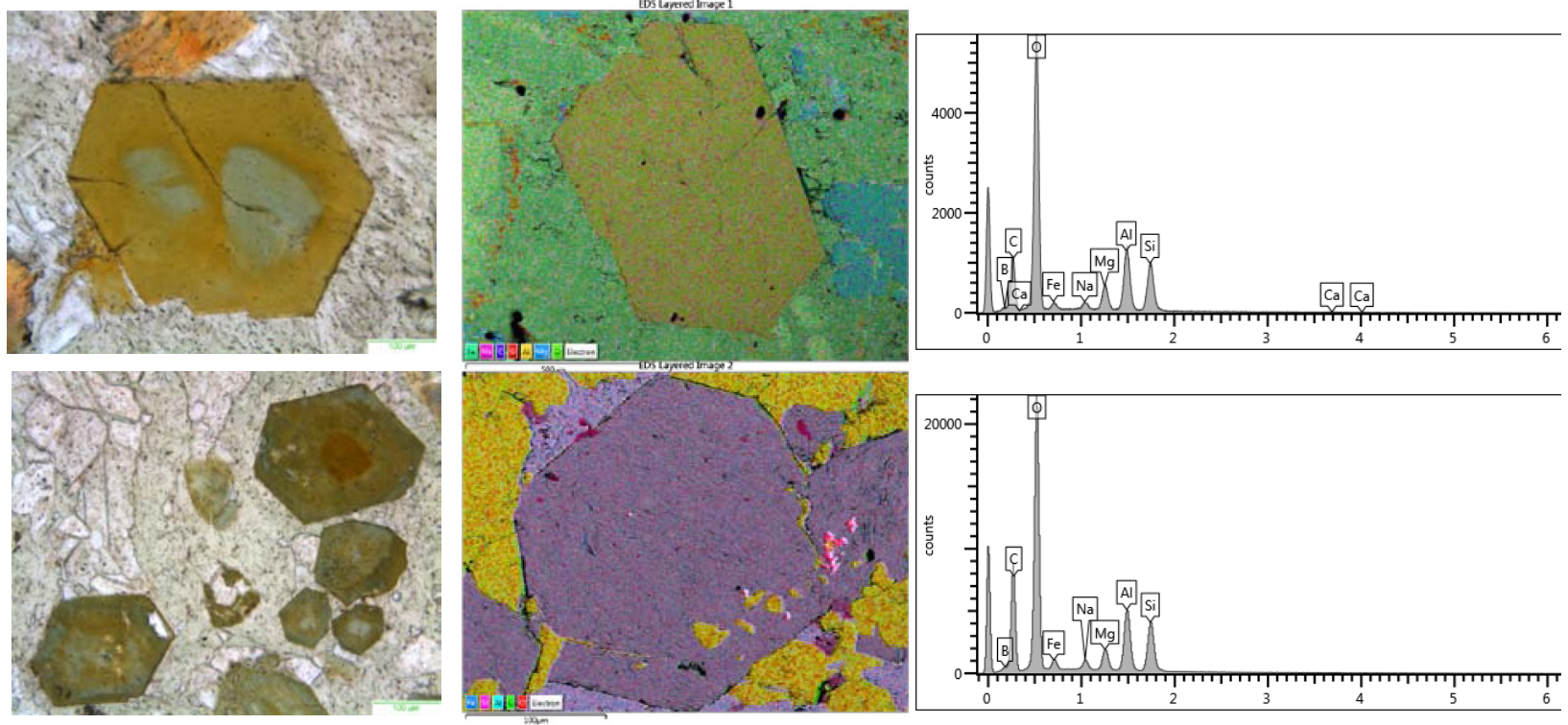

Figure 1: Left column are plane polarized optical microscope images of zoned tourmaline crystals. The center column is over-layered element maps and the right column is spectra from the cores of the crystals shown in the maps. 